MATHEMATICS OF COMPUTATION

Volume 74, Number 249 , Pages 177-200

S 0025-5718(04)01669-2

Article electronically published on July 22, 2004

\title{
MATHEMATICAL ANALYSIS OF ABSORBING BOUNDARY CONDITIONS FOR THE WAVE EQUATION: THE CORNER PROBLEM
}

\author{
OLIVIER VACUS
}

\begin{abstract}
Our goal in this work is to establish the existence and the uniqueness of a smooth solution to what we call in this paper the corner problem, that is to say, the wave equation together with absorbing conditions at two orthogonal boundaries. First we set the existence of a very smooth solution to this initial boundary value problem. Then we show the decay in time of energies of high order - higher than the order of the boundary conditions. This result shows that the corner problem is strongly well-posed in spaces smaller than in the half-plane case. Finally, specific corner conditions are derived to select the smooth solution among less regular solutions. These conditions are required to derive complete numerical schemes.
\end{abstract}

\section{INTRODUCTION}

1.1. The wave equation in the half-space. Let us consider the $2-\mathrm{D}$ wave equation on the half-space $\Omega_{o}=\left\{(x, y) \in \mathbb{R}^{2}, x \in \mathbb{R}, y<0\right\}$, with artificial boundary $\Gamma_{o}=\partial \Omega=\left\{(x, y) \in \mathbb{R}^{2}, x \in \mathbb{R}, y=0\right\}$. Associated with initial data $\left(u_{0}, u_{1}\right)$, this leads to the following initial boundary value problem:

$$
\begin{cases}\frac{\partial^{2} u}{\partial t^{2}}-\left(\frac{\partial^{2} u}{\partial x^{2}}+\frac{\partial^{2} u}{\partial y^{2}}\right)=0 & \text { on } \Omega_{o}, \forall t>0 \\ B_{N}^{y} u=0 & \text { on } \Gamma_{o}, \forall t>0, \\ u(x, y, t=0)=u_{0}(x, y) & \text { on } \Omega_{o}, \\ \frac{\partial u}{\partial t}(x, y, t=0)=u_{1}(x, y) & \text { on } \Omega_{o} .\end{cases}
$$

Here $\left(B_{N}^{y} u=0\right)$ is the absorbing boundary condition $(\mathrm{ABC}), B_{N}^{y}$ denotes the boundary operator: the index $y$ indicates that the outer normal is along $(O y)$, while the subscript $N$ gives the order of the operator.

Generally speaking, an $\mathrm{ABC}$ is defined following two different criteria.

First, as far as numerical simulations are concerned, $B_{N}^{y}$ must be chosen so that $u$ is as close as possible to $\bar{u}$, the solution to the wave equation on $\mathbb{R}^{2}$. In other words, $B_{N}^{y}$ must minimize the artificial reflections on $\Gamma_{o}$ : the more absorbing the $\mathrm{ABC}$ is, the smaller is the reflected energy back to $\Omega_{o}$. This issue will not be discussed in this paper (see [9] for a comprehensive presentation).

Received by the editor May 26, 2002 and, in revised form, June 29, 2003.

2000 Mathematics Subject Classification. Primary 78A40, 65N12; Secondary 65M12, 46N40.

Key words and phrases. Wave equation, absorbing boundary conditions, domain with a corner, energy methods, strong well-posedness, stability. 
Second, from the mathematical point of view, the stability of (1.1) is an interesting topic in itself. This issue is considered in this work in a special case.

Indeed, the analysis is restricted to specific local ABCs, derived from Padé's approximants (see [4] for instance). It is shown in [8] that, for $N=2 n$ or $2 n+1$, we have

$$
B_{N}^{y}=\left(\partial_{t}+\gamma \partial_{y}\right) \prod_{k=1}^{n}\left(\frac{\partial^{2}}{\partial t^{2}}+\alpha_{k} \frac{\partial^{2}}{\partial x^{2}}\right)-\partial_{t} \sum_{k=1}^{n} \beta_{k} \frac{\partial^{2}}{\partial x^{2}}\left[\prod_{j \neq k}\left(\frac{\partial^{2}}{\partial t^{2}}+\alpha_{j} \frac{\partial^{2}}{\partial x^{2}}\right)\right] .
$$

As explained in [9] where they are compared to more recent ABCs, these conditions are not the most efficient, but they give acceptable results for moderate time calculations. Besides, they have two major qualities.

First they can be used in variable coefficient cases. This is not possible with other conditions like the efficient ones presented in [10].

Second they are well adapted to the special shape of the artificial boundary like the planar boundary. Rectangular domains are considered in this paper, which can surround high aspect ratio obstacles like wires. With smooth artificial boundaries (see [5, 6], [12] or [10]), the computational domain can be much greater, which increases the cost of the interior solver.

The stability of Padé's approximants has been dealt with by Trefethen and Halpern in 16, and more recently by Ha Duong and Joly in [8]. Using an algebraic criterion in the first case and energy techniques in the second case, (1.1) is shown to be strongly well-posed if and only if the coefficients in (1.2) satisfy

$$
\left\{\begin{array}{l}
0 \leq \alpha_{1}<\alpha_{2}<\ldots<\alpha_{n}<1 \\
0 \leq \beta_{k}, 1 \leq k \leq n \\
\sum_{k=1}^{n} \frac{\beta_{k}}{1-\alpha_{k}}<\gamma .
\end{array}\right.
$$

The strong well-posedness of (1.1) (in the sense of Kreiss) means that there exists a solution $u$, that this solution is unique but also that there exists a norm of $u$ which can be controlled by the same norm applied to the initial data. As far as strong well-posedness is concerned, energy techniques are powerful tools. They have been adapted successfully to various cases, like curved boundaries ([18]) or the Helmholtz equation on a bounded domain with corners ([14]) for instance

The question is then to go from the half-space to bounded domains. Two ways are possible. As we said already, the first one consists of working with smooth artificial boundaries and will not be discussed here. The second one, more natural with cartesian coordinates, deals with several straight boundaries, as described below.

1.2. The corner problem. In this paper the propagation domain is reduced from the half-plane to the quarter-plane $\Omega=\left\{(x, y) \in \mathbb{R}^{2}, x<0, y<0\right\}$, as shown in Figure 1] The boundary $\Gamma=\partial \Omega$ is constituted by $\Gamma_{x}=\{x=0, y<0\}$, of outer normal along $(O x)$, and $\Gamma_{y}=\{x<0, y=0\}$, of outer normal along $(O y)$. This boundary admits a singularity, a "corner", at the origin $0(x=0, y=0)$. 


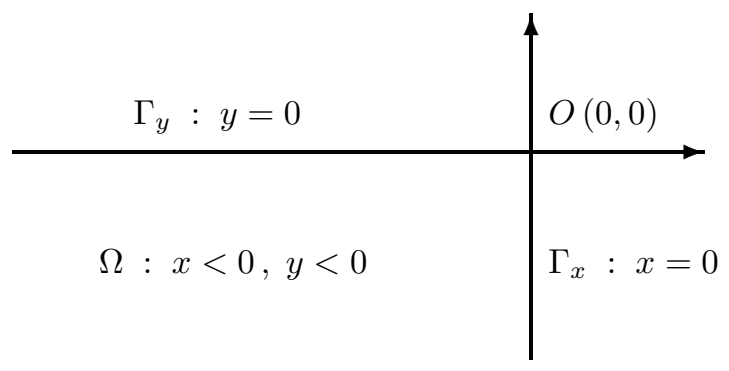

Figure 1. Definition of the calculus domain.

The $\mathrm{ABC}$ on $\Gamma_{y}$ is unchanged (see (1.2)), while on $\Gamma_{x}$ an equivalent condition is introduced (variables $x$ and $y$ are permuted):

$$
B_{N}^{x}=\left(\partial_{t}+\gamma \partial_{x}\right) \prod_{k=1}^{n}\left(\frac{\partial^{2}}{\partial t^{2}}+\alpha_{k} \frac{\partial^{2}}{\partial y^{2}}\right)-\partial_{t} \sum_{k=1}^{n} \beta_{k} \frac{\partial^{2}}{\partial y^{2}}\left[\prod_{j \neq k}\left(\frac{\partial^{2}}{\partial t^{2}}+\alpha_{j} \frac{\partial^{2}}{\partial y^{2}}\right)\right] .
$$

Given initial data $\left(u_{0}, u_{1}\right)$ such that

$$
\operatorname{Supp}\left(u_{0}, u_{1}\right) \subset \Omega,
$$

we can now define the corner problem as

$$
\begin{cases}\frac{\partial^{2} u}{\partial t^{2}}-\left(\frac{\partial^{2} u}{\partial x^{2}}+\frac{\partial^{2} u}{\partial y^{2}}\right)=0 & \text { on } \Omega, \forall t>0, \\ B_{N}^{x} u=0 & \text { on } \Gamma_{x}, \forall t>0, \\ B_{N}^{y} u=0 & \text { on } \Gamma_{y}, \forall t>0, \\ u(x, y, t=0)=u_{0}(x, y) & \text { on } \Omega, \\ \frac{\partial u}{\partial t}(x, y, t=0)=u_{1}(x, y) & \text { on } \Omega .\end{cases}
$$

This is the initial boundary value problem that we propose to analyze in this work. Following what was done in a half-plane, there are at least four issues to address, as soon as $N \geq 2$.

(Q1) Does there exist a solution (for regular data at least)?

(Q2) Is this solution unique?

(Q3) Is the stability equivalent in the half-plane and in the quarter-plane? (Or, in other words, are stable ABCs the same in both cases?)

(Q4) What would be the point of adding specific conditions at the corner, and how would we derive them?

As far as we know, all these issues remain open in the general case (for any $N)$. In 2] for instance, a similar problem (the $\mathrm{ABC}$ formulation is different) is considered but the emphasis is put on the numerical efficiency of the formulation rather than on the mathematical analysis. It would seem that only two papers contain relevant results.

First, in [1], a complete analysis is given for $N=2$, more precisely for the classical second order ABC (see subsection 2.1):

$$
\frac{\partial^{2} u}{\partial t^{2}}+\frac{\partial^{2} u}{\partial t \partial x}-\frac{1}{2} \frac{\partial^{2} u}{\partial y^{2}}=0 \text { on } \Gamma_{x} \quad \text { and } \quad \frac{\partial^{2} u}{\partial t^{2}}+\frac{\partial^{2} u}{\partial t \partial y}-\frac{1}{2} \frac{\partial^{2} u}{\partial x^{2}}=0 \text { on } \Gamma_{x} .
$$


The two main conclusions in this case are

- there exists a unique smooth solution to the corner problem;

- this smooth solution is the only one such that

$$
\frac{3}{2} \frac{\partial u}{\partial t}+\frac{\partial u}{\partial x}+\frac{\partial u}{\partial y}=0 \text { at } O .
$$

This last condition is a corner condition "derived by compatibility" (CCC): the computation of such a condition lies on the regularity of the solution. From the numerical point of view, it is shown that any other corner condition is worse because it generates a "corner wave" (a wave propagating backward inside $\Omega$ and centered at $O)$.

A second paper must be mentioned: in [17, Sheen adapted to the corner problem (this time again with $N=2$ ) the energy techniques introduced in [8] for the halfplane. The key point of his work shows that a more suitable energy in this case is of higher order (namely 3 ) than the ABC.

1.3. Outline of the paper. We propose to extend the main results presented in 1] and [17, so as to try to answer questions (Q1)-(Q4). Note that the main part of this work has been presented in [18].

The outline of the paper is as follows.

In Section 2, a smooth solution to the corner problem (1.6) is constructed for smooth initial data. The proof follows ideas contained in 11 and requires a specific assumption on the boundary operators $B_{N}^{x}$ and $B_{N}^{y}$. A brief overview of ABCs will show that this assumption is not a serious restriction.

In Section 3, we show the decay in time of an adapted "energy", that is to say, a quadratic form with respect to homogeneous differential operators applied to the smooth solution. Of course, this yields the uniqueness of this solution but more precisely this shows that the corner problem is strongly well-posed. The major difference with respect to the half-space case is that it is well-posed in a smaller space (roughly speaking $H^{2 N}$ instead of $H^{N}$; see subsection [3.2).

This explains why other less regular solutions to the corner problem may exist. The question then is to find a method to separate the smooth solution from the other ones.

In Section 4, we derive specific corner conditions, or CCC, which hold for the smooth solution only. Moreover, we show that, for any operators $B_{N}^{x}$ and $B_{N}^{y}$ of order $2 N$ or $2 N+1$, there always exist $2 N-1$ of such corner conditions. This is exactly the number of equations required to construct complete FDTD schemes.

\section{Existence of A SMOOTh SOlUtion to THE CORNER PROBlem}

Throughout this section, we assume that

$$
\left(u_{0}, u_{1}\right) \in \mathcal{D}(\Omega) \times \mathcal{D}(\Omega) .
$$

In subsection 2.1, we formulate precisely the required assumptions on the boundary operators and state the existence theorem. In subsection [2.2, we introduce two smooth functions, $v$ and $u$, solutions to two intermediate intial value problem easy to solve. In subsection [2.3, we just check that $u$ is indeed a solution to the corner problem. 
2.1. Statement of the result. In the general case, as recalled with (1.2), the boundary operator is any homogeneous differential operator involving even order derivatives with respect only to the tangential variable and fulfilling (1.3). Nevertheless, all these operators do not necessarily define efficient ABCs. The classical operators, often used in FDTD computations, are those introduced by Engquist and Majda in [4. They are derived from Padé's approximations (see [1], 9]), and can be recurcively defined on $\Gamma_{x}$ as

$$
\left\{\begin{array}{l}
B_{1}^{t, x}(u)=\frac{\partial u}{\partial t}+\frac{\partial u}{\partial x}=0 \\
B_{2}^{t, x}(u)=\frac{\partial^{2} u}{\partial t^{2}}+\frac{\partial^{2} u}{\partial t \partial x}-\frac{1}{2} \frac{\partial^{2} u}{\partial y^{2}}=0 \\
B_{N+1}^{t, x}(u) \partial_{t}\left(B_{N}^{t, x}(u)\right)-\frac{1}{4} \frac{\partial^{2}}{\partial y^{2}}\left(B_{N-1}^{t, x}(u)\right)=0, \forall N \geq 2
\end{array}\right.
$$

and, in the same way, on $\Gamma_{y}$, by interverting variables $x$ and $y$,

$$
\left\{\begin{array}{l}
B_{1}^{t, y}(u)=\frac{\partial u}{\partial t}+\frac{\partial u}{\partial y}=0 \\
B_{2}^{t, y}(u)=\frac{\partial^{2} u}{\partial t^{2}}+\frac{\partial^{2} u}{\partial t \partial y}-\frac{1}{2} \frac{\partial^{2} u}{\partial x^{2}}=0 \\
B_{N+1}^{t, y}(u)=\partial_{t}\left(B_{N}^{t, y}(u)\right)-\frac{1}{4} \frac{\partial^{2}}{\partial x^{2}}\left(B_{N-1}^{t, y}(u)\right)=0, \forall N \geq 2 .
\end{array}\right.
$$

A simpler approach is possible, which consists of using the following well-known property (see [8], [1], 11], 17]): if $u$ is a solution to the wave equation, then

$$
B_{N}^{x}(u)=\frac{1}{2^{N}}\left(\frac{\partial u}{\partial t}+\frac{\partial u}{\partial x}\right)^{N} \quad \text { and } \quad B_{N}^{x}(u)=\frac{1}{2^{N}}\left(\frac{\partial u}{\partial t}+\frac{\partial u}{\partial y}\right)^{N} .
$$

Our proof relies on this property. Consequently in this section, and only in this section, we do not deal with the general case: $B_{N}^{x}$ and $B_{N}^{y}$ are assumed to be of the same type as the "classical" boundary operators. More precisely, for a solution $u$ to the wave equation, we assume that there exist constants $C_{x}, C_{y}, a_{i}, b_{j}$ such that the boundary operators can be defined as

$$
B_{N}^{x}(u)=C_{x} \prod_{i=1}^{N}\left(\frac{\partial u}{\partial t}+a_{i} \frac{\partial u}{\partial x}\right) \quad \text { and } \quad B_{N}^{x}(u)=C_{y} \prod_{j=1}^{N}\left(\frac{\partial u}{\partial t}+b_{j} \frac{\partial u}{\partial y}\right)^{N} .
$$

This limitation still corresponds to a fairly wide class of ABCs including Engquist and Majda conditions. A further requirement concerns the operator coefficients $C_{x}, C_{y}$ and $a_{i}, b_{j}$ which must all be positive quantities:

$$
\mid \begin{aligned}
& C_{x} \geq 0, C_{y} \geq 0 \\
& a_{i} \geq 0, b_{j} \geq 0, \forall(i, j) \in\{1, \ldots, N\}
\end{aligned}
$$

We can now state the following theorem.

Theorem 2.1. Let $\left(u_{0}, u_{1}\right) \in \mathcal{D}(\Omega) \times \mathcal{D}(\Omega)$ with supports strictly included in $\Omega$. Assuming that assumptions (2.4) and (2.5) hold for the boundary operators, then there exists a smooth solution to the corner problem (1.6):

$$
u \in C^{\infty}\left(\mathbb{R}^{+}, \mathcal{D}(\Omega)\right) .
$$


2.2. Two intermediate problems. Let us first consider the wave equation on $\Omega$ with Dirichlet boundary conditions:

$$
\begin{cases}\frac{\partial^{2} v}{\partial t^{2}}-\frac{\partial^{2} v}{\partial x^{2}}-\frac{\partial^{2} v}{\partial y^{2}}=0 & \text { on } \Omega, \forall t \geq 0 \\ v=0 & \text { on } \Gamma, \forall t \geq 0\end{cases}
$$

It is well known that, for any initial data $\left(v_{0}(x, y), v_{1}(x, y)\right)$ in $\mathcal{D}(\Omega) \times \mathcal{D}(\Omega)$, with supports strictly included in $\Omega$, problem (2.6) admits a unique solution $v \in$ $C^{\infty}\left(\mathbb{R}^{+}, \mathcal{D}(\Omega)\right)$; this can be shown using for instance the theory of images. Let us carefully choose the initial data. The idea is to take

$$
v_{0}=B_{2 N}^{t, x, y}\left(u_{0}\right) \text { and } v_{1}=B_{2 N}^{t, x, y}\left(u_{1}\right),
$$

with

$$
B_{2 N}^{t, x, y}(u)=C_{x} C_{y} \prod_{i=1}^{N}\left(\partial_{t}+a_{i} \partial_{x}\right) \prod_{j=1}^{N}\left(\partial_{t}+b_{j} \partial_{y}\right)^{N} u,
$$

but, of course, this is only a formal definition. As a matter of fact, it is not possible to take time derivatives of functions $u_{0}$ and $u_{1}$. Nevertheless, it is fairly easy to overcome this difficulty. On the one hand, we can take

$$
\frac{\partial u_{0}}{\partial t}:=u_{1} \text {. }
$$

On the other hand, looking for solutions to the wave equation, it is obvious to take $u_{1}$ as

$$
\frac{\partial u_{1}}{\partial t}=\frac{\partial^{2} u_{0}}{\partial t^{2}}:=\Delta u_{0}
$$

A straightforward induction argument then leads to the general formula

$$
\left\{\begin{array}{l}
\frac{\partial^{2 k+1} u_{0}}{\partial t^{2 k+1}}=\frac{\partial^{2 k} u_{1}}{\partial t^{2 k}}=\Delta^{k} u_{1}, \\
\frac{\partial^{2 k+1} u_{1}}{\partial t^{2 k+1}}=\frac{\partial^{2 k+2} u_{0}}{\partial t^{2 k+2}}=\Delta^{k+1} u_{0} .
\end{array}\right.
$$

Eliminating the time derivatives in (2.7), this result enables us to define $v_{0}$ and $v_{1}$ as functions of $u_{0}$ and $u_{1}$ and their space derivatives. This completes the definition of a trivial Cauchy problem (2.6), whose unique solution is denoted $v$.

This function $v$ can now be used as data in a second initial value problem:

$$
\left\{\begin{array}{cl}
B_{2 N}^{t, x, y}(u)=v & \text { on } \Omega, \forall t \geq 0, \\
\frac{\partial^{2 k} u}{\partial t^{2 k}}(x, y, t=0)=\Delta^{k} u_{0} & \text { on } \Omega, 0 \leq k \leq N-1, \\
\frac{\partial^{2 k+1} u}{\partial t^{2 k+1}}(x, y, t=0)=\Delta^{k} u_{1} & \text { on } \Omega, 0 \leq k \leq N-1 .
\end{array}\right.
$$

Here, $\frac{\partial^{0} \varphi}{\partial t^{0}}$ as well as $\Delta^{0} \varphi$ is taken equal to $\varphi$. Thus the $2 N$ initial data of problem (2.9) are defined in such a way that $u$ must be a solution to the wave equation. In fact, as will be shown later, $u$ is a solution to (1.6).

Let us now check that $u$ is well defined, which is rather straightforward. For the same reasons as in [1], the Cauchy problem (2.9) is well-posed because we integrate in the quarter-plane $\Omega=\{x<0, y<0\}$ and coefficients $a_{i}$ and $b_{j}$ are all positive constants. Moreover this integration ensures that solution $u \in C^{\infty}\left(\mathbb{R}^{+}, \mathcal{D}(\Omega)\right)$. 
2.3. Proof of Theorem 2.1 As mentioned before, the proof reduces to checking that the function $u$ defined by (2.9) (and (2.6)) is a classical solution of problem (1.6).

Since the initial data $u(t=0)$ and $\frac{\partial u}{\partial t}(t=0)$ are clearly the same in (2.9) and (1.6), we just have to check that the wave equation on $\Omega$ and the boundary conditions on $\Gamma_{x}$ and $\Gamma_{y}$ are indeed satisfied.

First we introduce function $w=\frac{\partial^{2} u}{\partial t^{2}}-\Delta u$, and we show that it necessarily vanishes on $\Omega$. The first equation of set (2.6) implies that

$$
B_{2 N}^{t, x, y}(w)=0 \text { on } \Omega .
$$

Moreover, as $u_{0}$ is a solution to the wave equation, simple algebraic computations lead from (2.9) to

$$
0 \leq k \leq 2 N-1, \quad \frac{\partial^{k} w}{\partial t^{k}}(x, y, t=0)=0
$$

Equation (2.10), together with initial data (2.11), defines a trivial Cauchy problem which admits as unique solution the null function. In other words, we conclude that

$$
w=\frac{\partial^{2} u}{\partial t^{2}}-\Delta u=0
$$

As we can see, $u$ satisfies the wave equation on $\Omega$.

Following similar reasoning, we now consider

$$
w_{y}(x, y, t)=B_{N}^{y}(u(x, y, t)) \text { and } g_{y}(x, t)=w_{y}(x, 0, t) .
$$

From (2.9), we deduce that

$$
B_{N}^{x}\left(w_{y}\right)=v \text { on } \Omega, \forall t \geq 0 .
$$

Since we know that (see (2.6))

$$
v(x, 0, t)=0 \text { on } \Gamma_{y}, \forall t \geq 0,
$$

we deduce that $B_{N}^{x}\left(w_{y}\right)(x, 0, t)=0$ on $\Gamma_{y}, \forall t \geq 0$, that is to say,

$$
B_{N}^{x}\left(g_{y}\right)=0 \text { on } \Gamma_{y}, \forall t \geq 0 .
$$

Since the supports of the initial data of problem (1.6) are strictly included in $\Omega$, we can write the following relationship:

$$
g_{y}(x, t=0)=0 \text { and } \frac{\partial^{k} g_{y}}{\partial t^{k}}(x, t=0)=0,1 \leq k \leq N-1 .
$$

We then deduce from (2.16) and (2.17) that

$$
g_{y}(x, t=0)=B_{N}^{y}(u)(x, 0, t)=0 \text { on } \Gamma_{y}, \forall t \geq 0,
$$

which was our original goal. Permutating $x$ and $y$ in the previous computations suffices to reach equivalent conclusions on $\Gamma_{x}$, then completing the proof of Theorem 2.1 . 


\section{A PRIORI ESTIMATES}

We begin now the second part of this paper. As we have established in the previous section, there exists a very smooth solution $u$ to the corner problem, smooth enough to enable us to apply to $u$ operators of arbitrarily high order. This particular property is useful to apprehend the energy approach that we introduce in this section.

It is worth noting that, even though the existence result is obtained for a particular case, we make no specific assumption in this section concerning operators $B_{N}^{x}$ and $B_{N}^{y}$. The general case is treated.

In a first step, we briefly review the content of energy methods. In particular we summarize with the help of a few simple computations the energy result on which the work of Sheen (in the second order case) is based ([17). Then we present the case of the third order boundary conditions. This step is important in order to introduce the technical computations of subsection 3.2 in which the general case at $N$ order is treated.

3.1. About the energy method. As explained in [8, for any solution $v$ to the wave equation on $\Omega$, we have

$$
v\left(\frac{\partial^{2} v}{\partial t^{2}}-\Delta v\right)=0 .
$$

Integrating by parts and applying the Green formula, we then obtain

$$
\frac{1}{2} \frac{d}{d t}\left[\int_{\Omega}\left(\left|\frac{\partial v}{\partial t}\right|^{2}+|\nabla v|^{2}\right) d s\right]=\int_{\Gamma} \frac{\partial v}{\partial t} \frac{\partial v}{\partial n} d \gamma .
$$

In view of forthcoming computations, we now introduce the following notation:

$$
E(v)=\int_{\Omega}\left(\left|\frac{\partial v}{\partial t}\right|^{2}+|\nabla v|^{2}\right) d s .
$$

Now, since we are concerned with the constant-coefficient case, if $v$ is a smooth solution to the wave equation, so is any derivative of $v$. This explains why in our case, where $u$ is a $C^{\infty}$-function, identity (3.1) applies to every derivative of $u$.

The simplest case to illustrate how to use identity (3.1) is the case involving the first order condition:

$$
\frac{\partial u}{\partial t}+\frac{\partial u}{\partial n}=0 .
$$

In this case, we immediately have, from (3.1) applied to $u$,

$$
\frac{1}{2} \frac{d}{d t}\left[\int_{\Omega}\left(\left|\frac{\partial u}{\partial t}\right|^{2}+|\nabla u|^{2}\right) d s\right]=-\int_{\Gamma}\left|\frac{\partial u}{\partial t}\right|^{2} d \gamma=-\int_{\Gamma}\left|\frac{\partial u}{\partial n}\right|^{2} d \gamma,
$$

which leads to

$$
\|D u\|_{L^{\infty}\left(\mathbb{R}_{+}, L^{2}(\Omega)\right)} \leq\left\|u_{0}\right\|_{H^{1}(\Omega)}+\left\|u_{1}\right\|_{L^{2}(\Omega)} .
$$

(Here $D u$ denotes any first order derivative of $u$.) The analysis presented in [8] is based on a priori estimates similar to (3.4).

Remark 3.1. Due to the product before integrating, identity (3.1) is not "linear": it is not equivalent to applying it to $\alpha u$, or just to $u$ and then multiplying by $\alpha$. This will be useful in the energy computation. 
Let us now see what happens with a corner in the second order case. We present here a new way to lead the calculus presented in [17]. We remind the reader that the boundary conditions are then

$$
\frac{\partial^{2} u}{\partial t^{2}}+\frac{\partial^{2} u}{\partial t \partial x}-\frac{1}{2} \frac{\partial^{2} u}{\partial y^{2}}=0 \text { on } \Gamma_{x}, \quad \frac{\partial^{2} u}{\partial t^{2}}+\frac{\partial^{2} u}{\partial t \partial y}-\frac{1}{2} \frac{\partial^{2} u}{\partial x^{2}}=0 \text { on } \Gamma_{y} .
$$

First, we simply apply identity (3.1) to $\frac{\partial^{2} u}{\partial t^{2}}, \frac{\partial^{2} u}{\partial t \partial x}, \frac{\partial^{2} u}{\partial t \partial y}$ and $\frac{\partial^{2} u}{\partial x \partial y}$. We get

$$
\begin{aligned}
& \frac{d}{d t}\left(E\left(\frac{\partial^{2} u}{\partial t^{2}}\right)\right)=\int_{\Gamma_{y}} \frac{\partial^{3} u}{\partial t^{2} \partial y} \frac{\partial^{3} u}{\partial t^{3}} d x+\int_{\Gamma_{x}} \frac{\partial^{3} u}{\partial t^{2} \partial x} \frac{\partial^{3} u}{\partial t^{3}} d y \\
& \frac{d}{d t}\left(E\left(\frac{\partial^{2} u}{\partial t \partial x}\right)\right)=\int_{\Gamma_{y}} \frac{\partial^{3} u}{\partial t \partial x \partial y} \frac{\partial^{3} u}{\partial t^{2} \partial x} d x+\int_{\Gamma_{x}} \frac{\partial^{3} u}{\partial t \partial x^{2}} \frac{\partial^{3} u}{\partial t^{2} \partial x} d y \\
& \frac{d}{d t}\left(E\left(\frac{\partial^{2} u}{\partial t \partial y}\right)\right)=\int_{\Gamma_{y}} \frac{\partial^{3} u}{\partial t \partial y^{2}} \frac{\partial^{3} u}{\partial t^{2} \partial y} d x+\int_{\Gamma_{x}} \frac{\partial^{3} u}{\partial t \partial x \partial y} \frac{\partial^{3} u}{\partial t^{2} \partial y} d y \\
& \frac{d}{d t}\left(E\left(\frac{\partial^{2} u}{\partial x \partial y}\right)\right)=\int_{\Gamma_{y}} \frac{\partial^{3} u}{\partial x \partial y^{2}} \frac{\partial^{3} u}{\partial t \partial x \partial y} d x+\int_{\Gamma_{x}} \frac{\partial^{3} u}{\partial x^{2} \partial y} \frac{\partial^{3} u}{\partial t \partial x \partial y} d y
\end{aligned}
$$

We then add these four equation to end up with

$$
\begin{aligned}
& \frac{d}{d t}\left(E\left(\frac{\partial^{2} u}{\partial t^{2}}\right)+E\left(\frac{\partial^{2} u}{\partial t \partial x}\right)+E\left(\frac{\partial^{2} u}{\partial t \partial y}\right)+E\left(\frac{\partial^{2} u}{\partial x \partial y}\right)\right) \\
& =\int_{\Gamma_{y}}\left\{\frac{\partial^{3} u}{\partial t^{2} \partial y}\left(\frac{\partial^{3} u}{\partial t^{3}}+\frac{\partial^{3} u}{\partial t \partial y^{2}}\right)+\frac{\partial^{3} u}{\partial t \partial x \partial y}\left(\frac{\partial^{3} u}{\partial t^{2} \partial x}+\frac{\partial^{3} u}{\partial x \partial y^{2}}\right)\right\} d x \\
& \quad+\int_{\Gamma_{x}}\left\{\frac{\partial^{3} u}{\partial t^{2} \partial x}\left(\frac{\partial^{3} u}{\partial t^{3}}+\frac{\partial^{3} u}{\partial t \partial x^{2}}\right)+\frac{\partial^{3} u}{\partial t \partial x \partial y}\left(\frac{\partial^{3} u}{\partial t^{2} \partial y}+\frac{\partial^{3} u}{\partial x^{2} \partial y}\right)\right\} d y
\end{aligned}
$$

Concerning the boundary conditions (3.6), as mentioned before, we can also write, for any $u$ solution to the wave equation

$$
\frac{\partial^{2} u}{\partial t^{2}}+2 \frac{\partial^{2} u}{\partial t \partial y}+\frac{\partial^{2} u}{\partial y^{2}}=0 \text { on } \Gamma_{x}, \quad \frac{\partial^{2} u}{\partial t^{2}}+2 \frac{\partial^{2} u}{\partial t \partial x}+\frac{\partial^{2} u}{\partial x^{2}}=0 \text { on } \Gamma_{y} .
$$

The idea now consists of taking the derivative of the two previous conditions both with respect to time and space along the boundary:

$$
\text { on } \Gamma_{y}: \quad\left\{\begin{array}{l}
\frac{\partial^{3} u}{\partial t^{3}}+2 \frac{\partial^{3} u}{\partial t^{2} \partial y}+\frac{\partial^{3} u}{\partial t \partial y^{2}}=0 \\
\frac{\partial^{3} u}{\partial t^{2} \partial x}+2 \frac{\partial^{3} u}{\partial t \partial x \partial y}+\frac{\partial^{3} u}{\partial x \partial y^{2}}=0
\end{array}\right.
$$

and

$$
\text { on } \Gamma_{x}: \quad\left\{\begin{array}{l}
\frac{\partial^{3} u}{\partial t^{3}}+2 \frac{\partial^{3} u}{\partial t^{2} \partial x}+\frac{\partial^{3} u}{\partial t \partial x^{2}}=0 \\
\frac{\partial^{3} u}{\partial t^{2} \partial y}+2 \frac{\partial^{3} u}{\partial t \partial x \partial y}+\frac{\partial^{3} u}{\partial x^{2} \partial y}=0
\end{array}\right.
$$

One among different factorization possibilities on the boundary is

$$
\text { on } \Gamma_{y}: \quad\left\{\begin{array}{l}
-2 \frac{\partial^{3} u}{\partial t^{2} \partial y}=\frac{\partial^{3} u}{\partial t^{3}}+\frac{\partial^{3} u}{\partial t \partial y^{2}}, \\
-2 \frac{\partial^{3} u}{\partial t \partial x \partial y}=\frac{\partial^{3} u}{\partial t^{2} \partial x}+\frac{\partial^{3} u}{\partial x \partial y^{2}}
\end{array}\right.
$$


and

$$
\text { on } \Gamma_{x}: \quad\left\{\begin{array}{l}
-2 \frac{\partial^{3} u}{\partial t^{2} \partial x}=\frac{\partial^{3} u}{\partial t^{3}}+\frac{\partial^{3} u}{\partial t \partial x^{2}}, \\
-2 \frac{\partial^{3} u}{\partial t \partial x \partial y}=\frac{\partial^{3} u}{\partial t^{2} \partial y}+\frac{\partial^{3} u}{\partial x^{2} \partial y} .
\end{array}\right.
$$

We now see that plugging (3.9) and (3.10) into (3.7) leads to

$$
\begin{aligned}
\frac{d}{d t}\left[E\left(\frac{\partial^{2} u}{\partial t^{2}}\right)\right. & \left.+E\left(\frac{\partial^{2} u}{\partial t \partial x}\right)+E\left(\frac{\partial^{2} u}{\partial t \partial y}\right)+E\left(\frac{\partial^{2} u}{\partial x \partial y}\right)\right] \\
& =-2 \int_{\Gamma_{y}}\left|\frac{\partial^{3} u}{\partial t^{2} \partial y}\right|^{2} d x-2 \int_{\Gamma_{x}}\left|\frac{\partial^{3} u}{\partial t^{2} \partial x}\right|^{2} d y-4 \int_{\Gamma}\left|\frac{\partial^{3} u}{\partial t \partial x \partial y}\right|^{2} d \gamma .
\end{aligned}
$$

This shows that the quadratic form

$$
\begin{aligned}
\tilde{\mathcal{E}_{2}}(t)=\int_{\Omega}\left[\left\|\frac{\partial^{3} u}{\partial t^{3}}\right\|^{2}+2\left\|\frac{\partial^{3} u}{\partial t^{2} \partial x}\right\|^{2}+2\left\|\frac{\partial^{3} u}{\partial t^{2} \partial y}\right\|^{2}+3\left\|\frac{\partial^{3} u}{\partial t \partial x \partial y}\right\|^{2}\right. \\
\left.+\left\|\frac{\partial^{3} u}{\partial t \partial x^{2}}\right\|^{2}+\left\|\frac{\partial^{3} u}{\partial t \partial y^{2}}\right\|^{2}+\left\|\frac{\partial^{3} u}{\partial x^{2} \partial y}\right\|^{2}+\left\|\frac{\partial^{3} u}{\partial x \partial y^{2}}\right\|^{2}\right] d s
\end{aligned}
$$

is decreasing in time. We can now deduce the following theorem

Theorem 3.2. For $\left(u_{0}, u_{1}\right) \in \mathcal{D}(\Omega) \times \mathcal{D}(\Omega)$, there exists a unique solution to the corner problem (1.6) with boundary conditions (3.6) in $L^{\infty}\left(\mathbb{R}_{+}, H^{3}(\Omega)\right)$. Moreover,

$$
\left\|D^{3} u\right\|_{L^{\infty}\left(\mathbb{R}_{+}, L^{2}(\Omega)\right)} \leq 6\left(\left\|u_{0}\right\|_{H^{3}}+\left\|u_{1}\right\|_{H^{2}}\right) .
$$

Proof. A priori estimate (3.12) holds as soon as the energy $\tilde{\mathcal{E}}_{2}(t)$ is finite at time $t=0$. This gives the control of derivatives included in $\tilde{\mathcal{E}}_{2}(t)$ (see (3.11)). The control of $\frac{\partial^{3} u}{\partial x^{3}}$ and $\frac{\partial^{3} u}{\partial y^{3}}$ is obtained via the wave equation:

$$
\frac{\partial^{3} u}{\partial x^{3}}=\frac{\partial^{3} u}{\partial t^{2} \partial x}-\frac{\partial^{3} u}{\partial x \partial y^{2}}, \quad \frac{\partial^{3} u}{\partial y^{3}}=\frac{\partial^{3} u}{\partial t^{2} \partial y}-\frac{\partial^{3} u}{\partial x^{2} \partial y} .
$$

This completes the proof.

Remark 3.3. As mentioned in [8], it is also possible to get estimates of the trace of the solution on the boundary from (3.12). This point will not be discussed further in this article.

3.2. General case. In this section we show that a high order energy can be associated to $N$ order ABCs (like (1.2)) in the corner problem. For the sake of simplicity only the odd case is studied: $N=2 n+1$. For the even case, see [8] where minor differences are pointed out (in the half-plane case).

We propose the following theorem.

Theorem 3.4. For $\left(u_{0}, u_{1}\right) \in \mathcal{D}(\Omega) \times \mathcal{D}(\Omega)$, there exists a unique solution to the corner problem (1.6) with boundary conditions (1.2) and (1.4) in $L^{\infty}\left(\mathbb{R}_{+}, H^{4 n+1}(\Omega)\right)$. Moreover, $\exists C>0$, depending only on constants given in (1.3), such that the solution verifies

$$
\left\|D^{4 n+1} u\right\|_{L^{\infty}\left(\mathbb{R}_{+}, L^{2}(\Omega)\right)} \leq C\left(\left\|u_{0}\right\|_{H^{4 n+1}}+\left\|u_{1}\right\|_{H^{4 n}}\right) .
$$


3.2.1. Basic definitions. In the odd case, we have on $\Gamma_{y}$

$$
B_{N}^{t, y}=\left(\partial_{t}+\gamma \partial_{y}\right) \prod_{k=1}^{n}\left(\frac{\partial^{2}}{\partial t^{2}}-\alpha_{k} \frac{\partial^{2}}{\partial x^{2}}\right)-\partial_{t} \sum_{k=1}^{n} \beta_{k} \frac{\partial^{2}}{\partial x^{2}}\left[\prod_{j \neq k}^{n}\left(\frac{\partial^{2}}{\partial t^{2}}-\alpha_{j} \frac{\partial^{2}}{\partial x^{2}}\right)\right]=0
$$

with $\gamma,\left\{\beta_{k}\right\}_{k}$ and $\left\{\alpha_{k}\right\}_{k}$ some real coefficients such that assumption (1.3) holds. As far as solutions to the wave equations are considered, the boundary operator also reads

$$
\begin{aligned}
B_{N}^{t, y}=\left(\partial_{t}+\gamma \partial_{y}\right) & \prod_{k=1}^{n}\left(\left(1-\alpha_{k}\right) \frac{\partial^{2}}{\partial t^{2}}+\alpha_{k} \frac{\partial^{2}}{\partial y^{2}}\right) \\
& -\partial_{t} \sum_{k=1}^{n} \beta_{k}\left(\frac{\partial^{2}}{\partial t^{2}}-\frac{\partial^{2}}{\partial y^{2}}\right)\left[\prod_{j \neq k}^{n}\left(\left(1-\alpha_{j}\right) \frac{\partial^{2}}{\partial t^{2}}+\alpha_{j} \frac{\partial^{2}}{\partial y^{2}}\right)\right] .
\end{aligned}
$$

Following [8], we know that this can be written

$$
\frac{\partial Q^{y} u}{\partial y}+a \frac{\partial Q^{y} u}{\partial t}+\sum_{k=1}^{n} a_{k} \frac{\partial^{3} Q_{k}^{y} u}{\partial t \partial y^{2}}=0
$$

with the following notation

$$
\begin{gathered}
P_{k}^{y}=P_{k}\left(\partial_{t}, \partial_{y}\right)=\left(1-\alpha_{k}\right) \frac{\partial^{2}}{\partial t^{2}}+\alpha_{k} \frac{\partial^{2}}{\partial y^{2}} \\
Q^{y}=Q\left(\partial_{t}, \partial_{y}\right)=\prod_{k=1}^{n} P_{k}^{y}, \quad Q_{k}^{y}=Q_{k}\left(\partial_{t}, \partial_{y}\right)=\prod_{j \neq k}^{n} P_{j}^{y} .
\end{gathered}
$$

Similarly, for $\Gamma_{x}$, we can write

$$
\frac{\partial Q^{x} u}{\partial x}+a \frac{\partial Q^{x} u}{\partial t}+\sum_{k=1}^{n} a_{k} \frac{\partial^{3} Q_{k}^{x} u}{\partial t \partial x^{2}}=0
$$

with

$$
\mid \begin{gathered}
P_{k}^{x}=P_{k}\left(\partial_{t}, \partial_{x}\right)=\left(1-\alpha_{k}\right) \frac{\partial^{2}}{\partial t^{2}}+\alpha_{k} \frac{\partial^{2}}{\partial x^{2}} \\
Q^{x}=Q\left(\partial_{t}, \partial_{x}\right)=\prod_{k=1}^{n} P_{k}^{x}, \quad Q_{k}^{x}=Q_{k}\left(\partial_{t}, \partial_{x}\right)=\prod_{j \neq k}^{n} P_{j}^{x} .
\end{gathered}
$$

In these expressions, constants are assumed to be positive to ensure that the ABCs are stable (see assumption (1.3)):

$$
a_{k}=\frac{\beta_{k}}{1-\alpha_{k}} \geq 0 \quad \text { and } \quad a=\gamma-\sum_{k=1}^{n} \frac{\beta_{k}}{1-\alpha_{k}}=\gamma-\sum_{k=1}^{n} a_{k} \geq 0 .
$$

3.2.2. A priori estimates. Some elementary computations are now performed on $\Gamma_{y}$. From (3.14), we have

$$
\int_{\Gamma_{y}} \frac{\partial Q^{y} u}{\partial y} \cdot\left(-\frac{\partial Q^{y} u}{\partial y}\right) d x=\int_{\Gamma_{y}} \frac{\partial Q^{y} u}{\partial y}\left(a \frac{\partial Q^{y} u}{\partial t}+\sum_{k=1}^{n} a_{k} \frac{\partial^{3} Q_{k}^{y} u}{\partial t \partial y^{2}}\right) d x .
$$


Hence

$$
-\int_{\Gamma_{y}}\left|\frac{\partial Q^{y} u}{\partial y}\right|^{2} d x=\int_{\Gamma_{y}}\left(a \frac{\partial Q^{y} u}{\partial y} \frac{\partial Q^{y} u}{\partial t}+\sum_{k=1}^{n} a_{k} \frac{\partial Q^{y} u}{\partial y} \frac{\partial^{3} Q_{k}^{y} u}{\partial t \partial y^{2}}\right) d x .
$$

For any $k$, polynomial $Q^{y}$, and then $P_{k}^{y}$, can be expanded as

$$
\begin{aligned}
\frac{\partial Q^{y} u}{\partial y} \frac{\partial^{3} Q_{k}^{y} u}{\partial t \partial y^{2}}= & \partial_{y}\left(P_{k}\left(Q_{k} u\right)\right) \partial_{t}\left(\frac{\partial^{2} Q_{k}^{y} u}{\partial y^{2}}\right) \\
= & \alpha_{k} \partial_{t}\left(\frac{\partial^{2} Q_{k}^{y} u}{\partial y^{2}}\right) \partial_{y}\left(\frac{\partial^{2} Q_{k}^{y} u}{\partial y^{2}}\right) \\
& +\left(1-\alpha_{k}\right) \partial_{t}\left(\frac{\partial^{2} Q_{k}^{y} u}{\partial t \partial y}\right) \partial_{y}\left(\frac{\partial^{2} Q_{k}^{y} u}{\partial t \partial y}\right) .
\end{aligned}
$$

From (3.19) (and (3.18): $a_{k}\left(1-\alpha_{k}\right)=\beta_{k}$ ), we then deduce that

$$
\begin{aligned}
-\int_{\Gamma_{y}}\left|\frac{\partial Q^{y} u}{\partial y}\right|^{2}=\int_{\Gamma_{y}}\{a & \frac{\partial Q^{y} u}{\partial t} \frac{\partial Q^{y} u}{\partial y} \\
& +\sum_{k=1}^{n} a_{k} \alpha_{k} \partial_{t}\left(\frac{\partial^{2} Q_{k}^{y} u}{\partial y^{2}}\right) \partial_{y}\left(\frac{\partial^{2} Q_{k}^{y} u}{\partial y^{2}}\right) \\
& \left.+\sum_{k=1}^{n} \beta_{k} \partial_{t}\left(\frac{\partial^{2} Q_{k}^{y} u}{\partial t \partial y}\right) \partial_{y}\left(\frac{\partial^{2} Q_{k}^{y} u}{\partial t \partial y}\right)\right\} d x .
\end{aligned}
$$

Similarly, dealing with $\Gamma_{x}$, we get

$$
\begin{aligned}
-\int_{\Gamma_{x}}\left|\frac{\partial Q^{x} u}{\partial x}\right|^{2}=\int_{\Gamma_{x}}\{a & \frac{\partial Q^{x} u}{\partial t} \frac{\partial Q^{x} u}{\partial x} \\
& +\sum_{k=1}^{n} a_{k} \alpha_{k} \partial_{t}\left(\frac{\partial^{2} Q_{k}^{x} u}{\partial x^{2}}\right) \partial_{x}\left(\frac{\partial^{2} Q_{k}^{x} u}{\partial x^{2}}\right) \\
& \left.+\sum_{k=1}^{n} \beta_{k} \partial_{t}\left(\frac{\partial^{2} Q_{k}^{x} u}{\partial t \partial x}\right) \partial_{x}\left(\frac{\partial^{2} Q_{k}^{x} u}{\partial t \partial x}\right)\right\} d y .
\end{aligned}
$$

In identities (3.20) and (3.21), the key information is that the left-hand side is always negative. The idea then consists of writing the right-hand side as a derivative with respect to time and checking that the associated quantity is an "energy" (a definite positive form). As (3.20) and (3.21) are symmetrical with respect to $x$ and $y$, space derivatives on $\Gamma$ can be seen as normal derivatives. From (3.20) and (3.21) we introduce the high order operator

$$
\mathcal{Q}=\mathcal{Q}_{t, y} \mathcal{Q}_{t, x}
$$

with

$$
\mathcal{Q}_{t, y}=\left(a Q^{y}+\sum_{k=1}^{n} \beta_{k} \frac{\partial^{2} Q_{k}^{y}}{\partial t \partial y}+\sum_{k=1}^{n} a_{k} \alpha_{k} \frac{\partial^{2} Q_{k}^{y}}{\partial y^{2}}\right)
$$

and

$$
\mathcal{Q}_{t, x}=\left(a Q^{x}+\sum_{k=1}^{n} \beta_{k} \frac{\partial^{2} Q_{k}^{x}}{\partial t \partial x}+\sum_{k=1}^{n} a_{k} \alpha_{k} \frac{\partial^{2} Q_{k}^{x}}{\partial x^{2}}\right) .
$$


Operator $\mathcal{Q}$ can be expanded as

$$
\begin{aligned}
\mathcal{Q}=a^{2} Q^{x} Q^{y} & +\sum_{k=1}^{n} a \beta_{k} Q^{y} \frac{\partial^{2} Q_{k}^{x}}{\partial t \partial x}+\sum_{k=1}^{n} a a_{k} \alpha_{k} Q^{x} \frac{\partial^{2} Q_{k}^{y}}{\partial y^{2}}+\sum_{k=1}^{n} a \beta_{k} Q^{x} \frac{\partial^{2} Q_{k}^{y}}{\partial t \partial y} \\
& +\sum_{k=1}^{n} \sum_{k^{\prime}=1}^{n} \beta_{k} \beta_{k^{\prime}} \frac{\partial^{2} Q_{k^{\prime}}^{x}}{\partial t \partial x} \frac{\partial^{2} Q_{k}^{y}}{\partial t \partial y}+\sum_{k=1}^{n} \sum_{k^{\prime}=1}^{n} a_{k^{\prime}} \alpha_{k^{\prime}} \beta_{k} \frac{\partial^{2} Q_{k}^{y}}{\partial t \partial y} \frac{\partial^{2} Q_{k^{\prime}}^{x}}{\partial x^{2}} \\
& +\sum_{k=1}^{n} a a_{k} \alpha_{k} Q^{x} \frac{\partial^{2} Q_{k}^{y}}{\partial y^{2}}+\sum_{k=1}^{n} \sum_{k^{\prime}=1}^{n} a_{k} \alpha_{k} \beta_{k^{\prime}} \frac{\partial^{2} Q_{k^{\prime}}^{x}}{\partial t \partial x} \frac{\partial^{2} Q_{k}^{y}}{\partial y^{2}} \\
& +\sum_{k=1}^{n} \sum_{k^{\prime}=1}^{n} a_{k} \alpha_{k} a_{k^{\prime}} \alpha_{k^{\prime}} \frac{\partial^{2} Q_{k^{\prime}}^{x}}{\partial x^{2}} \frac{\partial^{2} Q_{k}^{y}}{\partial y^{2}} .
\end{aligned}
$$

Identity (1.6) must then be successively applied to each term of the expansion (there are exactly $(2 n+1)^{2}$ terms). For instance, for the first term, we get

$$
\frac{1}{2} \frac{d}{d t}\left[E\left(Q^{x} Q^{y}\right)\right]=\int_{\Gamma} \partial_{t}\left(Q^{x} Q^{y} u\right) \partial_{n}\left(Q^{x} Q^{y} u\right)
$$

which yields

$$
\frac{1}{2} \frac{d}{d t}\left[a^{2} E\left(Q^{x} Q^{y}\right)\right]=a^{2} \int_{\Gamma_{y}} \frac{\partial Q^{y}}{\partial y}\left(Q^{x} u\right) \frac{\partial Q^{y}}{\partial t}\left(Q^{x} u\right)+a^{2} \int_{\Gamma_{x}} \frac{\partial Q^{x}}{\partial x}\left(Q^{y} u\right) \frac{\partial Q^{x}}{\partial t}\left(Q^{y} u\right) .
$$

After summing all the terms with the correct associated factor, we get

$$
\frac{d}{d t}(\mathcal{E}(u))=\int_{\Gamma_{y}} A_{x} d x+\int_{\Gamma_{y}} A_{y} d y
$$

On the left-hand side of (3.23), $\mathcal{E}$ denotes the appropriate high order $(4 n+1)$ energy

$$
\begin{aligned}
\mathcal{E}(u)= & a^{2} E\left(Q^{x} Q^{y} u\right)+\sum_{k=1}^{n} a \beta_{k} E\left(Q^{y} \frac{\partial^{2} Q_{k}^{x} u}{\partial t \partial x}\right)+\sum_{k=1}^{n} a a_{k} \alpha_{k} E\left(Q^{x} \frac{\partial^{2} Q_{k}^{y} u}{\partial y^{2}}\right) \\
& +\sum_{k=1}^{n} a \beta_{k} E\left(Q^{x} \frac{\partial^{2} Q_{k}^{y} u}{\partial t \partial y}\right)+\sum_{k=1}^{n} \sum_{k^{\prime}=1}^{n} \beta_{k} \beta_{k^{\prime}} E\left(\frac{\partial^{2} Q_{k^{\prime}}^{x}}{\partial t \partial x} \frac{\partial^{2} Q_{k}^{y} u}{\partial t \partial y}\right) \\
& +\sum_{k=1}^{n} \sum_{k^{\prime}=1}^{n} a_{k^{\prime}} \alpha_{k^{\prime}} \beta_{k} E\left(\frac{\partial^{2} Q_{k}^{y}}{\partial t \partial y} \frac{\partial^{2} Q_{k^{\prime}}^{x} u}{\partial x^{2}}\right) \\
& +\sum_{k=1}^{n} a a_{k} \alpha_{k} E\left(Q^{x} \frac{\partial^{2} Q_{k}^{y} u}{\partial y^{2}}\right)+\sum_{k=1}^{n} \sum_{k^{\prime}=1}^{n} a_{k} \alpha_{k} \beta_{k^{\prime}} E\left(\frac{\partial^{2} Q_{k^{\prime}}^{x}}{\partial t \partial x} \frac{\partial^{2} Q_{k}^{y} u}{\partial y^{2}}\right) \\
& +\sum_{k=1}^{n} \sum_{k^{\prime}=1}^{n} a_{k} \alpha_{k} a_{k^{\prime}} \alpha_{k^{\prime}} E\left(\frac{\partial^{2} Q_{k^{\prime}}^{x}}{\partial x^{2}} \frac{\partial^{2} Q_{k}^{y} u}{\partial y^{2}}\right) .
\end{aligned}
$$

It is allowable to call $\mathcal{E}(u)$ an energy as long as we deal with stable ABCs: constants $a, \beta_{k} \alpha_{k}$ and $a_{k}$ are then all positive quantities (see (3.18)). 
On the right hand side of (3.23), two boundary quantities, $A_{x}$ on $\Gamma_{x}$ and $A_{y}$ on $\Gamma_{y}$, have been introduced:

$$
\begin{aligned}
& A_{x}=a^{2} \frac{\partial Q^{y}}{\partial y}\left(Q^{x} u\right) \frac{\partial Q^{y}}{\partial t}\left(Q^{x} u\right) \\
& +\sum_{k=1}^{n} a \beta_{k} \frac{\partial^{3} Q_{k}^{y}}{\partial t \partial y^{2}}\left(Q^{x} u\right) \frac{\partial^{3} Q_{k}^{y}}{\partial t^{2} \partial y}\left(Q^{x} u\right)+\sum_{k=1}^{n} a \beta_{k} \frac{\partial Q^{y}}{\partial y}\left(\frac{\partial^{2} Q_{k}^{x} u}{\partial t \partial x}\right) \frac{\partial Q^{y}}{\partial t}\left(\frac{\partial^{2} Q_{k}^{x} u}{\partial t \partial x}\right) \\
& +\sum_{k=1}^{n=1} a a_{k} \alpha_{k} \frac{\partial^{3} Q_{k}^{y}}{\partial y^{3}}\left(Q^{x} u\right) \frac{\partial^{3} Q_{k}^{y}}{\partial t \partial y^{2}}\left(Q^{x} u\right)+\sum_{k=1}^{n} a a_{k} \alpha_{k} \frac{\partial Q^{y}}{\partial y}\left(\frac{\partial^{2} Q_{k}^{x} u}{\partial x^{2}}\right) \frac{\partial Q^{y}}{\partial t}\left(\frac{\partial^{2} Q_{k}^{x} u}{\partial x^{2}}\right) \\
& +\sum_{k=1}^{n} \sum_{k^{\prime}=1}^{n} \beta_{k} \beta_{k^{\prime}} \frac{\partial^{3} Q_{k}^{y}}{\partial t \partial y^{2}}\left(\frac{\partial^{2} Q_{k^{\prime}}^{x} u}{\partial t \partial x}\right) \frac{\partial^{3} Q_{k}^{y}}{\partial t^{2} \partial y}\left(\frac{\partial^{2} Q_{k^{\prime}}^{x} u}{\partial t \partial x}\right) \\
& +\sum_{k=1}^{n} \sum_{k^{\prime}=1}^{n} a_{k^{\prime}} \alpha_{k^{\prime}} \beta_{k} \frac{\partial^{3} Q_{k}^{y}}{\partial y^{3}}\left(\frac{\partial^{2} Q_{k^{\prime}}^{x} u}{\partial t \partial x}\right) \frac{\partial^{3} Q_{k}^{y}}{\partial t \partial y^{2}}\left(\frac{\partial^{2} Q_{k^{\prime}}^{x} u}{\partial t \partial x}\right) \\
& +\sum_{k=1}^{n} \sum_{k^{\prime}=1}^{n} a_{k} \alpha_{k} \beta_{k^{\prime}} \frac{\partial^{3} Q_{k}^{y}}{\partial t \partial y^{2}}\left(\frac{\partial^{2} Q_{k^{\prime}}^{x} u}{\partial x^{2}}\right) \frac{\partial^{3} Q_{k}^{y}}{\partial t^{2} \partial y}\left(\frac{\partial^{2} Q_{k^{\prime}}^{x} u}{\partial x^{2}}\right) \\
& +\sum_{k=1}^{n} \sum_{k^{\prime}=1}^{n} a_{k} \alpha_{k} a_{k^{\prime}} \alpha_{k^{\prime}} \frac{\partial^{3} Q_{k}^{y}}{\partial y^{3}}\left(\frac{\partial^{2} Q_{k^{\prime}}^{x} u}{\partial x^{2}}\right) \frac{\partial^{3} Q_{k}^{y}}{\partial t \partial y^{2}}\left(\frac{\partial^{2} Q_{k^{\prime}}^{x} u}{\partial x^{2}}\right)
\end{aligned}
$$

and

$$
\begin{aligned}
& A_{y}=a^{2} \frac{\partial Q^{x}}{\partial x}\left(Q^{y} u\right) \frac{\partial Q^{x}}{\partial t}\left(Q^{y} u\right) \\
& +\sum_{k=1}^{n} a \beta_{k} \frac{\partial^{3} Q_{k}^{x}}{\partial t \partial x^{2}}\left(Q^{y} u\right) \frac{\partial^{3} Q_{k}^{x}}{\partial t^{2} \partial x}\left(Q^{y} u\right)+\sum_{k=1}^{n} a \beta_{k} \frac{\partial Q^{x}}{\partial x}\left(\frac{\partial^{2} Q_{k}^{y} u}{\partial t \partial y}\right) \frac{\partial Q^{x}}{\partial t}\left(\frac{\partial^{2} Q_{k}^{y} u}{\partial t \partial y}\right) \\
& +\sum_{k=1}^{n} a a_{k} \alpha_{k} \frac{\partial^{3} Q_{k}^{x}}{\partial x^{3}}\left(Q^{y} u\right) \frac{\partial^{3} Q_{k}^{x}}{\partial t \partial x^{2}}\left(Q^{y} u\right)+\sum_{k=1}^{n} a a_{k} \alpha_{k} \frac{\partial Q^{x}}{\partial x}\left(\frac{\partial^{2} Q_{k}^{y} u}{\partial y^{2}}\right) \frac{\partial Q^{x}}{\partial t}\left(\frac{\partial^{2} Q_{k}^{y} u}{\partial y^{2}}\right) \\
& +\sum_{k=1}^{n} \sum_{k^{\prime}=1}^{n} \beta_{k} \beta_{k^{\prime}} \frac{\partial^{3} Q_{k}^{x}}{\partial t \partial x^{2}}\left(\frac{\partial^{2} Q_{k^{\prime}}^{y} u}{\partial t \partial y}\right) \frac{\partial^{3} Q_{k}^{x}}{\partial t^{2} \partial x}\left(\frac{\partial^{2} Q_{k^{\prime}}^{y} u}{\partial t \partial y}\right) \\
& +\sum_{k=1}^{n} \sum_{k^{\prime}=1}^{n} a_{k^{\prime}} \alpha_{k^{\prime}} \beta_{k} \frac{\partial^{3} Q_{k}^{x}}{\partial x^{3}}\left(\frac{\partial^{2} Q_{k^{\prime}}^{y} u}{\partial t \partial y}\right) \frac{\partial^{3} Q_{k}^{x}}{\partial t \partial x^{2}}\left(\frac{\partial^{2} Q_{k^{\prime}}^{y} u}{\partial t \partial y}\right) \\
& +\sum_{k=1}^{n} \sum_{k^{\prime}=1}^{n} a_{k} \alpha_{k} \beta_{k^{\prime}} \frac{\partial^{3} Q_{k}^{x}}{\partial t \partial x^{2}}\left(\frac{\partial^{2} Q_{k^{\prime}}^{y} u}{\partial y^{2}}\right) \frac{\partial^{3} Q_{k}^{x}}{\partial t^{2} \partial x}\left(\frac{\partial^{2} Q_{k^{\prime}}^{y} u}{\partial y^{2}}\right) \\
& +\sum_{k=1}^{n} \sum_{k^{\prime}=1}^{n} a_{k} \alpha_{k} a_{k^{\prime}} \alpha_{k^{\prime}} \frac{\partial^{3} Q_{k}^{x}}{\partial x^{3}}\left(\frac{\partial^{2} Q_{k^{\prime}}^{y} u}{\partial y^{2}}\right) \frac{\partial^{3} Q_{k}^{x}}{\partial t \partial x^{2}}\left(\frac{\partial^{2} Q_{k^{\prime}}^{y} u}{\partial y^{2}}\right) .
\end{aligned}
$$

It now remains to perform the adequate factorization on each boundary. Let us denote

$$
\begin{array}{r}
F_{y}(W)=a \frac{\partial Q^{y}}{\partial y}(W) \frac{\partial Q^{y}}{\partial t}(W)+\sum_{k=1}^{n} \beta_{k} \frac{\partial^{3} Q_{k}^{y}}{\partial t \partial y^{2}}(W) \frac{\partial^{3} Q_{k}^{y}}{\partial t^{2} \partial y}(W) \\
+\sum_{k=1}^{n} a_{k} \alpha_{k} \frac{\partial^{3} Q_{k}^{y}}{\partial y^{3}}(W) \frac{\partial^{3} Q_{k}^{y}}{\partial t \partial y^{2}}(W)
\end{array}
$$


and

$$
\begin{aligned}
F_{x}(W)=a \frac{\partial Q^{x}}{\partial x}(W) \frac{\partial Q^{x}}{\partial t}(W)+\sum_{k=1}^{n} \beta_{k} \frac{\partial^{3} Q_{k}^{x}}{\partial t \partial x^{2}}(W) \frac{\partial^{3} Q_{k}^{x}}{\partial t^{2} \partial x}(W) \\
+\sum_{k=1}^{n} a_{k} \alpha_{k} \frac{\partial^{3} Q_{k}^{x}}{\partial x^{3}}(W) \frac{\partial^{3} Q_{k}^{x}}{\partial t \partial x^{2}}(W) .
\end{aligned}
$$

We find

$$
A_{y}=a F_{y}\left(Q^{x} u\right)+\sum_{k^{\prime}=1}^{n} \beta_{k^{\prime}} F_{y}\left(\frac{\partial^{2} Q_{k^{\prime}}^{x} u}{\partial t \partial x}\right)+\sum_{k^{\prime}=1}^{n} a_{k^{\prime}} \alpha_{k^{\prime}} F_{y}\left(\frac{\partial^{2} Q_{k^{\prime}}^{x} u}{\partial x^{2}}\right)
$$

and

$$
A_{x}=a F_{x}\left(Q^{y} u\right)+\sum_{k^{\prime}=1}^{n} \beta_{k^{\prime}} F_{x}\left(\frac{\partial^{2} Q_{k^{\prime}}^{y} u}{\partial t \partial y}\right)+\sum_{k^{\prime}=1}^{n} a_{k^{\prime}} \alpha_{k^{\prime}} F_{x}\left(\frac{\partial^{2} Q_{k^{\prime}}^{y} u}{\partial y^{2}}\right) .
$$

But, (3.20) reads

$$
-\int_{\Gamma_{y}}\left|\frac{\partial Q^{y} u}{\partial y}\right|^{2}=\int_{\Gamma_{y}} F_{y}(u) d x
$$

and (3.21) reads

$$
-\int_{\Gamma_{y}}\left|\frac{\partial Q^{y} u}{\partial y}\right|^{2}=\int_{\Gamma_{y}} F_{x}(u) d x
$$

So, using $(2(2 n+1)$ times) expansions (3.20) and (3.21), we get

$$
\begin{aligned}
& \quad \frac{d \mathcal{E}(u)}{d t}= \\
& -\int_{\Gamma_{y}}\left(a\left|\frac{\partial Q^{y}}{\partial y}\left(Q^{x} u\right)\right|^{2}+\sum_{k=1}^{n} \beta_{k}\left|\frac{\partial Q^{y}}{\partial y}\left(\frac{\partial^{2} Q_{k}^{x}}{\partial t \partial x}\right)\right|^{2}+\sum_{k=1}^{n} a_{k} \alpha_{k}\left|\frac{\partial Q^{y}}{\partial y}\left(\frac{\partial^{2} Q_{k}^{x}}{\partial x^{2}}\right)\right|^{2}\right) d x \\
& -\int_{\Gamma_{x}}\left(a\left|\frac{\partial Q^{x}}{\partial x}\left(Q^{y} u\right)\right|^{2}+\sum_{k=1}^{n} \beta_{k}\left|\frac{\partial Q^{x}}{\partial x}\left(\frac{\partial^{2} Q_{k}^{y}}{\partial t \partial y}\right)\right|^{2}+\sum_{k=1}^{n} a_{k} \alpha_{k}\left|\frac{\partial Q^{x}}{\partial x}\left(\frac{\partial^{2} Q_{k}^{y}}{\partial y^{2}}\right)\right|^{2}\right) d y .
\end{aligned}
$$

This shows the decay in time of the energy $\mathcal{E}(u)$, which is the key to the proof.

Remark 3.5. In the previous computations, it is assumed that the $\mathrm{ABC}$ on $\Gamma_{y}$ holds for $Q^{x} u, \frac{\partial^{2} Q_{k}^{x} u}{\partial t \partial x}$ and $\frac{\partial^{2} Q_{k}^{x} u}{\partial x^{2}}$; this is true because $u$ is regular and because these three operators are defined with time and tangential derivatives only. The same conclusion is true on $\Gamma_{x}$ for $Q^{y} u, \frac{\partial^{2} Q^{x} u}{\partial t \partial y}$ and $\frac{\partial^{2} Q^{x} u}{\partial y^{2}}$.

3.2.3. Conclusion of the proof of Theorem 3.4. To complete the proof of Theorem [3.4, it remains to see that the control of the energy yields the control of any $4 n+1$ order derivative. To do this, we show how to control any (first) derivative of any $4 n$ order derivative.

Indeed, the definition of $\mathcal{E}(u)$ shows that any first derivative of $v \in V$ is uniformly bounded in time, where

$$
\begin{aligned}
& V=\left\{Q^{x} Q^{y},\left\{Q^{y} \frac{\partial^{2} Q_{k}^{x} u}{\partial t \partial x}\right\}_{k},\left\{Q^{x} \frac{\partial^{2} Q_{k}^{y} u}{\partial y^{2}}\right\}_{k},\left\{Q^{x} \frac{\partial^{2} Q_{k}^{y} u}{\partial t \partial y} u\right\}_{k},\left\{\frac{\partial^{2} Q_{k^{\prime}}^{x}}{\partial t \partial x} \frac{\partial^{2} Q_{k}^{y} u}{\partial t \partial y}\right\}_{k, k^{\prime}}\right. \\
& \left.\left\{\frac{\partial^{2} Q_{k}^{y}}{\partial t \partial y} \frac{\partial^{2} Q_{k^{\prime}}^{x} u}{\partial x^{2}}\right\}_{k, k^{\prime}},\left\{Q^{y} \frac{\partial^{2} Q_{k}^{y} u}{\partial x^{2}}\right\}_{k},\left\{\frac{\partial^{2} Q_{k^{\prime}}^{x}}{\partial t \partial x} \frac{\partial^{2} Q_{k}^{y} u}{\partial y^{2}}\right\}_{k, k^{\prime}},\left\{\frac{\partial^{2} Q_{k^{\prime}}^{x}}{\partial x^{2}} \frac{\partial^{2} Q_{k}^{y} u}{\partial y^{2}}\right\}_{k, k^{\prime}}\right\}^{\prime} .
\end{aligned}
$$


So, let us check that every polynomial

$$
s^{T} \xi_{x}^{X} \xi_{y}^{Y} \quad \text { with } \mid \begin{aligned}
& X+Y+T=4 n \\
& X \leq 2 n, Y \leq 2 n
\end{aligned}
$$

is a linear combination of elements of the following set:

$$
\begin{aligned}
V_{s, \xi_{x}, \xi_{y}}= & \left\{Q\left(s, \xi_{x}\right) Q\left(s, \xi_{y}\right),\left\{s \xi_{x} Q_{k}\left(s, \xi_{x}\right) Q\left(s, \xi_{y}\right)\right\}_{k},\left\{s \xi_{y} Q_{k}\left(s, \xi_{y}\right) Q\left(s, \xi_{x}\right)\right\}_{k}\right. \\
& \left\{\xi_{x}^{2} Q_{k}\left(s, \xi_{x}\right) Q\left(s, \xi_{y}\right)\right\}_{k},\left\{\xi_{y}^{2} Q_{k}\left(s, \xi_{y}\right) Q\left(s, \xi_{x}\right)\right\}_{k} \\
& \left\{s^{2} \xi_{x} \xi_{y} Q_{k}\left(s, \xi_{x}\right) Q_{k^{\prime}}\left(s, \xi_{y}\right)\right\}_{k, k^{\prime}},\left\{s \xi_{x}^{2} \xi_{y} Q_{k}\left(s, \xi_{x}\right) Q_{k^{\prime}}\left(s, \xi_{y}\right)\right\}_{k, k^{\prime}} \\
& \left.\left\{s \xi_{x} \xi_{y}^{2} Q_{k}\left(s, \xi_{x}\right) Q_{k^{\prime}}\left(s, \xi_{y}\right)\right\}_{k, k^{\prime}},\left\{\xi_{x}^{2} \xi_{y}^{2} Q_{k}\left(s, \xi_{x}\right) Q_{k^{\prime}}\left(s, \xi_{y}\right)\right\}_{k, k^{\prime}}\right\}
\end{aligned}
$$

This will ensure the result, because, when the order of derivatives with respect to $x$ or $y$ is greater than $2 n$, it can always be reduced by use of the wave equation (as we did in the previous section for $N=3$ ).

Now, with $T=T_{x}+T_{y}$ such that $T_{x}+X=2 n=T_{y}+Y$, we can factorize $s^{T} \xi_{x}^{X} \xi_{y}^{Y}=\left(s^{T_{x}} \xi_{x}^{X}\right)\left(s^{T_{y}} \xi_{y}^{Y}\right)$ and use the following lemma (see [8]).

Lemma 3.6. Let $\mathcal{P}_{2 n}$ denote the set of polynomials of two variables, $P(s, \xi)$, which are homogeneous and of degree $2 n$. Then, using polynomials introduced in (3.15) and (3.17),

$$
\left\{Q(s, \xi),\left\{s^{2} Q_{k}(s, \xi)\right\}_{1 \leq k \leq n},\left\{s \xi Q_{k}(s, \xi)\right\}_{1 \leq k \leq n}\right\}
$$

is a basis of $\mathcal{P}_{2 n}$.

This lemma guarantees that $\left(s^{T_{x}} \xi_{x}^{X}\right)$ is a linear combination of

$$
Q\left(s, \xi_{x}\right),\left\{s^{2} Q_{k}\left(s, \xi_{x}\right)\right\}_{1 \leq k \leq n},\left\{s \xi_{x} Q_{k}\left(s, \xi_{x}\right)\right\}_{1 \leq k \leq n},
$$

and $\left(s^{T_{y}} \xi_{y}^{Y}\right)$ is a linear combination of

$$
Q\left(s, \xi_{y}\right),\left\{s^{2} Q_{k}\left(s, \xi_{y}\right)\right\}_{1 \leq k \leq n},\left\{s \xi_{y} Q_{k}\left(s, \xi_{y}\right)\right\}_{1 \leq k \leq n},
$$

hence the result by taking the product.

\section{Corner compatibility conditions (CCC)}

At this point, we know that there exists a smooth solution $u$ to the corner problem (1.6); we shall denote it by $u_{\infty}$ in this section. We also know that this solution is unique in $H^{4 n+1}$. The trouble is that this space can be much smaller than $H^{2 n+1}$, which is the "natural" space where solutions are to be found. It is thus possible, we can even say that it is likely, that there exist other solutions, less regular than $u_{\infty}$.

A rigorous proof of this assertion is given in [1] for $N=2$. It is interesting to recall in this case that the difference between the regular solution and the others can be interpreted as a spurious reflexion at the corner, and numerical tests (see Figure 2) illustrate the impact of the corner condition on the quality of the absorption. This gives a good reason to modify the definition of the corner problem (1.6) in order to eliminate corner waves, at least in computations.

To do so, new boundary conditions, and more precisely corner conditions (since they hold at the corner only), must be introduced. Such corner conditions can be obtained by means of linear combinations of the initial equations and their derivatives. This is why they hold if and only if the solution is regular enough; otherwise derivatives would be meaningless. 

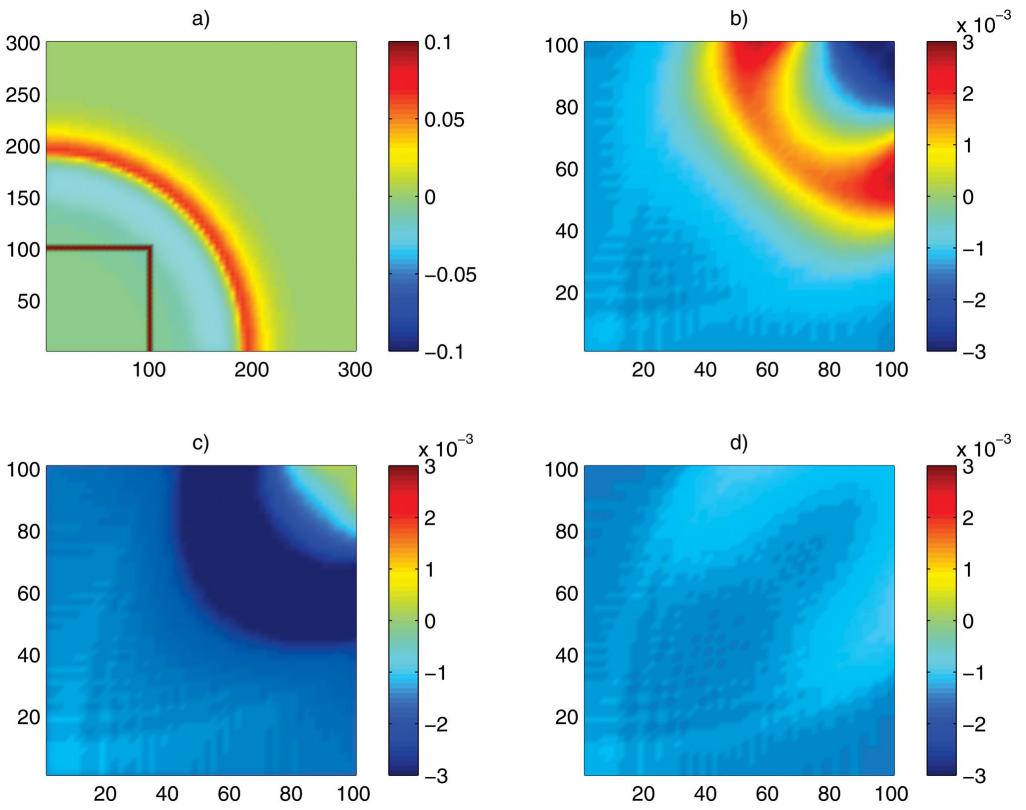

Figure 2. Exact discrete solution (a) computed on a large $300 \times$ 300 domain and three discrete solutions computed on a $100 \times 100$ domain and different corner conditions: optimal condition $\left(C C C_{2}\right)$ (d) (see (4.1) page 195), while in the other two cases the factor $3 / 2$ is either increased to 2 (in (b)) or decreased to 1 (in (c)).

Before we proceed to see how to derive corner conditions, it is fairly easy to understand why we have to introduce them and how many corner conditions are expected. The first way is analytical. Let us consider an $\mathrm{ABC}$ of order $2 n$ or $2 n+1$. If this $\mathrm{ABC}$ is seen as an ordinary differential equation (ODE) with tangential variable $s$ ( $y$ on $\Gamma_{x}$ and $x$ on $\Gamma_{y}$ ), the order of this ODE is $2 n$. Both boundaries $\Gamma_{x}$ and $\Gamma_{y}$ admit a "boundary", at the corner. This means that the solution is well defined if, and only if, at the corner, $2 n$ "boundary" conditions are specified, both on $\Gamma_{x}$ and $\Gamma_{y}$. These conditions can involve $u$, its derivatives with respect to time and its derivatives with respect to the tangential variable on each boundary. If nothing is done, there exists only one such condition: the continuity of the solution yields $u(x \rightarrow 0, y=0)=u(x=0, y \rightarrow 0)$. This shows that $2 n-1$ conditions are missing.

The second way to reach the same conclusion uses the same ideas in association this time with a numerical approach. Indeed, from the equations of problem (1.6) alone, it is not possible to derive a complete numerical scheme. Let us consider for instance the case of a FDTD scheme, as described in Figure 3, If interior (resp. exterior) nodes can be evaluated using the discrete wave equation (resp. the discrete $\mathrm{ABC})$, it turns out that no relationship applies at the corner.

In this sequel, we propose to work with the following definition of the corner conditions. 


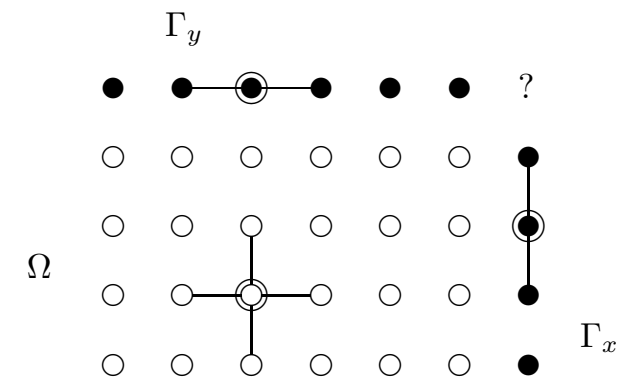

FiguRE 3. FDTD scheme: nodes and discrete laplacian operators within $\Omega$ and on $\Gamma$; the solution cannot be computed at the corner.

Definition 4.1. The equation $C_{M} u=0$ at the corner $O$ is a corner condition for problem (1.6) if, and only if, the operator $C$ is a homogeneous differential operator of order $M$, such that $1 \leq M<N(N$ denoting the order of the $\mathrm{ABC}$ on $\Gamma)$.

In this work we deal more precisely with corner compatibility conditions (CCC).

Definition 4.2. A corner condition is said to be compatible if, and only if, it holds for $u_{\infty}$ :

$$
C_{M} u_{\infty}(x=0, y=0, t)=0, \forall t \geq 0 .
$$

We can now state the following result.

Conjecture 4.3. In problem (1.6) with $\mathrm{ABC}$ of order $N=2 n$ or $N=2 n+1$, there exist exactly $2 n-1$ corner compatibility conditions (CCC).

We did not achieve establishing this result in the general case, but an algorithm is proposed below to determine for any given $N$ these (CCC). The idea of the computation simply consists of mixing the equations of the corner problem so as to eliminate the pure spatial operator. It is then possible to get low order conditions, namely the (CCC), by integrating in time.

4.1. An algebraic formalism. The key point of the computation is to transpose the mathematical analysis into a linear algebraic framework. To make this possible, the notation has to be adapted. From now on the differential operators will be seen as polynomials:

$$
\frac{\partial^{N} u}{\partial t^{T} \partial x^{X} \partial y^{Y}} \rightarrow s^{T} \xi_{x}^{X} \xi_{y}^{Y} \quad \text { with } X+Y+T=N .
$$

Thus the ABC can be viewed as a homogeneous polynomial of $N$ degree. This polynomial belongs to a space of dimension $N(N+1)$, whose canonical basis $\left\{s^{T} \xi_{x}^{X} \xi_{y}^{Y}\right\}_{T, X, Y}$ must be ordered so as to ensure the uniqueness of the algebraic definition:

(1) first with respect to increasing $T$;

(2) then with respect to increasing $|X-Y|$;

(3) lastly with respect to increasing $Y$. 
This gives $\left\{\xi_{x} \xi_{y}, \xi_{x}^{2}, \xi_{y}^{2}, s \xi_{x}, s \xi_{y}, s^{2}\right\}$ for $N=2$,

$$
\left\{\xi_{x}^{2} \xi_{y}, \xi_{x} \xi_{y}^{2}, \xi_{x}^{3}, \xi_{y}^{3}, s \xi_{x} \xi_{y}, s \xi_{x}^{2}, s \xi_{y}^{2}, s^{2} \xi_{x}, s^{2} \xi_{y}, s^{3}\right\}
$$

for $N=3$, and so on.

Of course, multiplications by $s$ (resp. $\xi_{x}$ and $\xi_{y}$ ) stands for derivatives with respect to $t$ (resp. $x$ and $y$ ).

4.1.1. $N=2$, classical case. For $N=2$, the representation of the ABCs on $\Gamma_{x}$ and $\Gamma_{y}$ are reduced to

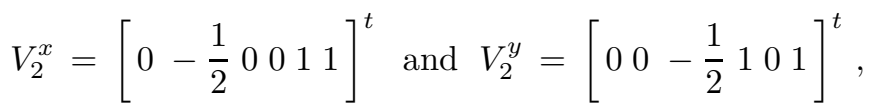

associated with a wave equation $W=\left[\begin{array}{llllll}0 & -1 & -1 & 0 & 0 & 1\end{array}\right]^{t}$. Let us now introduce the matrix $M=\left[W V_{2}^{x} V_{2}^{y}\right]$. This $6 \times 3$ matrix can be divided into two square matrices:

$$
M=\left[\begin{array}{l}
M_{1} \\
M_{2}
\end{array}\right], \text { with } M_{1}=\left[\begin{array}{ccc}
0 & 0 & 0 \\
-1 & -\frac{1}{2} & 0 \\
-1 & 0 & -\frac{1}{2}
\end{array}\right] .
$$

Let $X \in \operatorname{Ker} M_{1}$. Due to ordering as described above, any such vector $X$ gives a linear combination of the three initial equations, where all the pure spatial derivatives vanish. Here, we see that $\operatorname{dim}\left(\operatorname{Ker} M_{1}\right)=1$, and $X=[2-1-1]^{t}$. Thus, a (CCC) is given by $M_{2} X=\left[\begin{array}{lll}3 & 2 & 2\end{array}\right]^{t}$, that is to say,

$$
3 s^{2}+2 s \xi_{x}+2 s \xi_{y}=0 .
$$

Only terms with factor $s$ remain (this is due to the homogeneity of the ABC operator), which can be eliminated; this simply means that the (CCC) is integrated in time. Finally we get

$$
\left(C C C_{2}\right) \quad: \quad \frac{3}{2} \frac{\partial u}{\partial t}+\frac{\partial u}{\partial x}+\frac{\partial u}{\partial y}=0,
$$

the same condition as given in [1.

It now remains to repeat this two-step algorithm in higher order cases:

- define $M_{1}$ and $M_{2}$ from the wave equation and the ABCs;

- find $X \in \operatorname{Ker} M_{1}$ and $X \notin \operatorname{Ker} M_{2}$ to get $M_{2} X$ as a (CCC).

(Indeed, if $X \in \operatorname{Ker} M_{2}$, the (CCC) would reduce to $0=0$.)

4.1.2. $N=2$, general case. The above computation relies on the fact that matrix $M_{1}$ is singular. Let us check that this is always true, whatever the ABCs are. Let us assume that now

$$
V_{2}^{x}=\left[\begin{array}{llllll}
0 & a_{x} & 0 & 0 & b_{x} & 1
\end{array}\right]^{t} \text { and } V_{2}^{y}=\left[\begin{array}{llllll}
0 & 0 & a_{y} & b_{y} & 0 & 1
\end{array}\right]^{t} .
$$

In this case, $M_{1}$ and $M_{2}$ read

$$
M_{1}=\left[\begin{array}{ccc}
0 & 0 & 0 \\
-1 & a_{x} & 0 \\
-1 & 0 & a_{y}
\end{array}\right] \text { and } M_{2}=\left[\begin{array}{ccc}
0 & 0 & 0 \\
-1 & a_{x} & 0 \\
-1 & 0 & a_{y}
\end{array}\right] \text {. }
$$

The Kern of $M_{1}$ is never empty, and there always exists a (CCC): $\left[11 / a_{x} 1 / a_{y}\right]^{t}$. Of course, $a_{x}$ (resp. $a_{y}$ ) is assumed to be different; otherwise, the ABC can be integrated in time and is not of order $N$.

In the sequel, Padé's ABCs are considered for the sake of clarity, but generalization is nevertheless possible. 
4.1.3. $N=3$. The ABCs are now given by

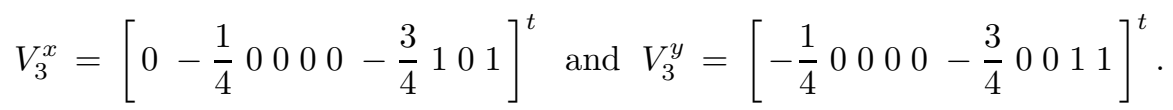

To mimic the previous computation, it is necessary to "increase" the order of the wave equation by taking the $x$ - and $y$-derivatives:

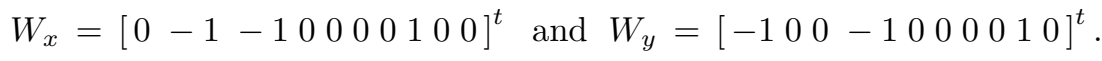

A $9 \times 4$ matrix can now be introduced: $M=\left[W_{x} W_{y} V_{3}^{y} V_{3}^{x}\right]$. Once again, this matrix can be seen as $M=\left[M_{1} M 2\right]^{t}$, where $M_{1}$ is a $(4 \times 4)$ square matrix:

$$
M_{1}=\left[\begin{array}{cccc}
0 & -1 & -\frac{1}{4} & 0 \\
-1 & 0 & 0 & -\frac{1}{4} \\
-1 & 0 & 0 & 0 \\
0 & -1 & 0 & 0
\end{array}\right] \text {. }
$$

The difficulty here is that $M_{1}$ is invertible ( $\operatorname{det} M_{1}=\frac{1}{16}$ ). This does not mean that there is no (CCC), but simply that the computation must be performed at a higher order. Indeed, the wave equation can be derived once more; with the $x$ - and the $t$-derivatives of $B_{3}^{t, y}$ on $\Gamma_{y}$ on the one hand and with the $y$ - and the $t$-derivatives of $B_{3}^{t, x}$ on $\Gamma_{y} x$ on the other hand, we get a $15 \times 9$ matrix

$$
M=\left[W_{x y} W_{x^{2}} W_{y^{2}}\left(V_{3}^{y}\right)_{x}\left(V_{3}^{x}\right)_{y} W_{t x} W_{t y}\left(V_{3}^{y}\right)_{t}\left(V_{3}^{x}\right)_{t}\right],
$$

and the upper block is now

$$
M_{1}=\left[\begin{array}{ccccccccc}
0 & -1 & -1 & 0 & 0 & 0 & 0 & 0 & 0 \\
-1 & 0 & 0 & -\frac{1}{4} & 0 & 0 & 0 & 0 & 0 \\
-1 & 0 & 0 & 0 & -\frac{1}{4} & 0 & 0 & 0 & 0 \\
0 & -1 & 0 & 0 & 0 & 0 & 0 & 0 & 0 \\
0 & 0 & -1 & 0 & 0 & 0 & 0 & 0 & 0 \\
0 & 0 & 0 & 0 & 0 & 0 & -1 & -\frac{1}{4} & 0 \\
0 & 0 & 0 & 0 & 0 & -1 & 0 & 0 & -\frac{1}{4} \\
0 & 0 & 0 & -\frac{3}{4} & 0 & -1 & 0 & 0 & 0 \\
0 & 0 & 0 & 0 & -\frac{3}{4} & 0 & -1 & 0 & 0
\end{array}\right]
$$

Now, $\operatorname{dim}\left(\operatorname{Ker} M_{1}\right)=1$, and we have

$$
X=\left[\begin{array}{lllllllll}
3 & 0 & 0 & -12 & -12 & 9 & 9 & -4 & -4
\end{array}\right] .
$$

Finally this leads to the following (CCC) for $N=3$ :

$$
\left(C C C_{3}\right): 24 \frac{\partial^{2} u}{\partial t^{2}}+13\left(\frac{\partial^{2} u}{\partial t \partial x}+\frac{\partial^{2} u}{\partial t \partial y}\right)+7 \frac{\partial^{2} u}{\partial x \partial y}-9\left(\frac{\partial^{2} u}{\partial x^{2}}+\frac{\partial^{2} u}{\partial y^{2}}\right)=0 .
$$

This result was first explicated in [1, after simplification by the wave equation

$$
15 \frac{\partial^{2} u}{\partial t^{2}}+13\left(\frac{\partial^{2} u}{\partial t \partial x}+\frac{\partial^{2} u}{\partial t \partial y}\right)+7 \frac{\partial^{2} u}{\partial x \partial y}=0 .
$$

These two technical points, high order computation and wave equation factorization, are now discussed in the general case. 


\subsection{About Conjecture 4.3.}

4.2.1. Necessary and sufficient order of the (CCC) computation. First of all, it must be recalled that, if every derivative of the wave equation may be used in the computation, only tangential $-x$ on $\Gamma_{y}$ and $y$ on $\Gamma_{x}$-and time derivatives of the ABCs are allowable.

Then, it must be understood why, with these derivatives, high order computation can be performed; here "high order" means greater than $N$, the ABCs order. The main reason lies in the perfect matching between the number of equations to be handled on the one hand (the initial equations and their derivatives) and the number of unknowns to be eliminated on the other hand.

Let $N$ be the ABCs order and $\nu$ the computation order; of course, $\nu \geq N$.

When $\nu=N$, there are $\nu+1$ (or $N+1$ ) unknowns to be eliminated: it is the dimension of the space of two uknown homogeneous polynomials. There are also $\nu+1$ equations: two $\mathrm{ABCs}$ and $\nu-1$ derivatives of the wave equation.

The key point lies in the linearity of these relationships: it explains why results are conserved when $\nu$ is increased. Let $k \geq 0$. From $\nu+k$ to $\nu+k+1$, there are $(\nu+k+1)+1$ new unknowns, and

- two new equations from the ABCs (tangential derivatives);

- $(\nu+k-1)+1$ new equations from the wave equation (spatial derivatives from order 2 to order $\nu+k+1$ ).

The result is proved by induction; $\forall N \geq 2, \forall \nu \geq N$, it guarantees that matrix $M_{1}^{N, \nu}$ is a square matrix of dimension $\sum_{k=N}^{\nu}(k+1)$.

4.2.2. Kern of $M_{1}^{N, \nu}$. Matrix $M_{1}^{N, \nu}$ is singular as soon as $\nu>N$. More precisely, we can state

Lemma 4.4. $\forall N \geq 2, \forall \nu \geq N$, the dimension of the kern of $M_{1}^{N, \nu}$ is given by

$$
\operatorname{dim}\left(\operatorname{Ker} M_{1}^{N, \nu}\right)= \begin{cases}E\left(\frac{\nu-N+1}{2}\right) & \text { if } N \text { is odd, } \\ E\left(\frac{\nu-N}{2}\right)+1 & \text { if } N \text { is even. }\end{cases}
$$

(Here, $E(\cdot)$ is the floor function.)

This result is important: it gives the number of (CCC) which can be derived from the corner problem.

Proof. Surprisingly, the proof is rather simple. With the algebraic formalism introduced above, matrix $M_{1}^{N, \nu}$ is block triangular. The blocks on the diagonal are all square, with size $m$ increasing from $N+1$ to $\nu+1$. Moreover, these blocks share the same shape:

$$
\Delta_{m}^{a_{x}, a_{y}}=\left[\begin{array}{ccccccccc}
0 & -1 & -1 & 0 & . & . & . & . & 0 \\
-1 & 0 & 0 & -1 & 0 & & & & . \\
-1 & 0 & 0 & . & -1 & 0 & & & . \\
0 & -1 & & . & & . & & & . \\
. & 0 & . & & . & & & & . \\
. & & & & & . & . & a_{x} & 0 \\
. & & & 0 & -1 & & . & . & a_{y} \\
. & & & & 0 & -1 & . & 0 & 0 \\
0 & . & . & . & . & 0 & -1 & 0 & 0
\end{array}\right] .
$$


The first columns come from the wave equation $((\nu+k-1)+1$ columns, as explained above) while the two last ones come the ABCs. An elementary determinant computation, as developed in Appendix A, shows that a block of this type is singular if and only if its size is odd. Moreover, when it is singular, $\operatorname{dim}\left(\operatorname{Ker} \Delta_{m}^{a, b}\right)=1$. This completes the proof of Lemma 4.4 the dimension of the kern is exactly the number of odd size diagonal blocks.

4.2.3. From $X_{i} \in \operatorname{Ker} M_{1}^{N, \nu}$ to $(C C C)$. At this point, we have seen why it is possible to perform computations at any order greater than $N$ and that increasing $\nu$ provides one time out of two new eigenvectors $X_{i} \in \operatorname{Ker} M_{1}^{N, \nu}$. It remains to say that each eigenvector does not necessarily gives a (CCC). This is due to the special structure of $M_{2}$, which is only filled with zeros when $\nu$ increases:

$$
M_{2}^{N, N+k+1}=\left[\begin{array}{lll}
0 & \mid & M_{2}^{N, N+k}
\end{array}\right] \quad, \forall k \geq 0 .
$$

As a consequence, $X_{i} \in \operatorname{Ker} M_{1}^{N, \nu}$ with $i$ large is such that $X_{i} \in \operatorname{Ker} M_{2}^{N, \nu}$ too; it then gives no new $(\mathrm{CCC})$ but just $0=0$. The last question to be studied is to determine how many vectors are such that

$$
X_{i} \in \operatorname{Ker} M_{1}^{N, \nu} \quad \text { and } \quad X_{i} \notin \operatorname{Ker} M_{2}^{N, \nu} .
$$

Exact computation in the general case would be tedious. Roughly speaking, it depends on the position and the numbers of nonzero elements in $M_{1}^{N, \nu}$. For the ABCs, $N+1$ blocks contain such elements, and thus, $X_{i} \in \operatorname{Ker} M_{2}^{N, \nu}$ for $\nu \simeq 3 N$. This yields $N_{C C C} \simeq N$, when numerical testing gives $N_{C C C}=2 n-1$ for $N=2 n$ or $N=2 n+1$. Exact computations (with integer coefficients) are possible with MAPLE up to $N=20$.

\section{Conclusion}

In this paper, we have first extended the classical well-posedness results (in the sense of Kreiss) for the wave equation to the corner problem, a case with two orthogonal boundaries. In particular, this shows that stable absorbing conditions are the same on a straightline boundary and on a boundary with a right angle corner. The method, based on energy estimates, also applies to rectangular domains, which are typically used as computational domains.

In a second part, we have defined specific corner conditions (corner compatibility conditions) and explained why they are required to construct efficient numerical schemes. In the general case with respect to $N$, the boundary condition's order, we have given the exact number of expected (and necessary) corner conditions, and we indicated how they can be derived from the other equations of the initial problem.

Different further works are now possible. On the one hand, as mentioned in [9], it would be of interest to extend the "planar boundary" error estimates to the corner case and to assess their long time behaviour. Numerical tests must also be completed to check whether or not the mathematical stability yields the numerical stability for $N \geq 3$. On the other hand, the case of different corners (with angle not necessarily right) is of major practical interest and should be assessed as a matter of priority. 


\section{APPENDIX A: KeRn of $\Delta_{m}^{a_{x}, a_{y}}$}

In this appendix, we only show that $\Delta_{m}^{a_{x}, a_{y}}$ is singular if and only if $m$ is odd by computing $\operatorname{det}\left(\Delta_{m}^{a_{x}, a_{y}}\right)$, but a characteristic polynomial computation is also possible and would give more complete results.

First, $\Delta_{m}^{a_{x}, a_{y}}$ is computed for $2 \leq m \leq 5$ and $(a, b) \in \mathbb{R}^{2}$ :

$$
\begin{aligned}
\operatorname{det}\left(\Delta_{2}^{a_{x}, a_{y}}\right)=\left|\begin{array}{cc}
0 & -1 \\
-1 & 0
\end{array}\right| & =-1, \quad \operatorname{det}\left(\Delta_{3}^{a_{x}, a_{y}}\right)=\left|\begin{array}{ccc}
0 & -1 & a_{x} \\
-1 & 0 & 0 \\
-1 & 0 & 0
\end{array}\right|=0, \\
& \operatorname{det}\left(\Delta_{4}^{a_{x}, a_{y}}\right)=\left|\begin{array}{cccc}
0 & -1 & a_{x} & 0 \\
-1 & 0 & 0 & a_{y} \\
-1 & 0 & 0 & 0 \\
0 & -1 & 0 & 0
\end{array}\right|=a_{x} a_{y}, \\
& \operatorname{det}\left(\Delta_{5}^{a_{x}, a_{y}}\right)=\left|\begin{array}{ccccc}
0 & -1 & -1 & 0 & 0 \\
-1 & 0 & 0 & a_{x} & 0 \\
-1 & 0 & 0 & 0 & a_{y} \\
0 & -1 & 0 & 0 & 0 \\
0 & 0 & -1 & 0 & 0
\end{array}\right|=0 .
\end{aligned}
$$

(As we said in Section $4 a_{x} a_{y} \neq 0$.) Then, in any case, we see that there is only one nonzero coefficient in the last two lines and the last two columns of $\Delta_{m}^{a_{x}, a_{y}}$. Thus, for $m \geq 6$, a direct expansion leads to

$$
\operatorname{det}\left(\Delta_{m}^{a_{x}, a_{y}}\right)=a_{x} a_{y} \operatorname{det}\left(\Delta_{m-4}^{-1,-1}\right),
$$

hence the result, by induction.

\section{REFERENCES}

1. A. Bamberger, P. Joly and J.E. Roberts, Second order absorbing boundary conditions for the wave equation: a solution for the corner problem, in SIAM J. Numer. Anal., 27 (1990), pp. 323-352. MR 91b:35066

2. F. Collino, High order absorbing boundary conditions for wave propagation models: Straight line boundary and corner cases, Second international conference on mathematical and numerical aspects of wave propagation, SIAM, pp. 161-171, 1995. MR.94e:65091

3. F. Collino, Conditions absorbantes d'ordre élevé pour des modèles de propagation d'onde, technical report 1970, INRIA, 1993.

4. B. Engquist and A. Majda, Absorbing boundary conditions for the numerical simulation of waves, Math. Comp., 31 (1977), pp. 629-651. MF 55:9555

5. D. Givoli, Non-reflecting boundary conditions, J. Comput. Phys., 94 (1991), pp. 1-29. MR92a:65266

6. D. Givoli, Exact representations on artificial interfaces and applications in mechanics, Applied Mechanics Review, 52 (1999), pp. 333-349.

7. B. Gustafson, H.O. Kreiss and A. Sundstrom, Stability theory of difference approximations for mixed initial boundary value problems (II), Math. Comp., 26 (1972), pp. 649-686. MR 49:6634

8. T. Ha Duong and P. Joly, On the stability analysis of boundary conditions for the wave equation by energy methods. Part 1: The homogeneous case, Math. Comp., 62 (1994), pp. 539563. MR94k:35174

9. T. Hagstrom, Radiation boundary conditions for the numerical simulation of waves, Acta Numerica, Cambridge University Press (1999), pp. 47-106. MR2002c:35171

10. B. Alpert, L. Greengard, T. Hagstrom, Nonreflecting Boundary conditions for the timedependent wave equation, J. Comp. Phys., 180 (2002), pp. 270-296. MR2003e:65140

11. R.L. Higdon, Absorbing boundary conditions for difference approximations to the multidimensional wave equation, Math. Comp., 47 (1977), pp. 437-459. MR87m:65131 
12. S. Tsynkov, Numerical solution of problems on unbounded domains. A review, Appl. Numer. Math., 27 (1998), pp. 465-532. MR99i:65116

13. P. Joly, O. Vacus, Stabilité de conditions aux limites pour l'équation des ondes par des méthodes énergétiques: le cas des bords courbes, INRIA Rocquencourt technical report 2849, France, 1996.

14. P. Joly, S. Lorhengel, O. Vacus, Un résultat d'existence et d'unicité pour l'équation de Helmholtz avec conditions aux limites absorbantes d'ordre 2, Note aux Comptes Rendus de l'Académie des Sciences, t. 329 (3), pp. 193-198, 1999. MR2000e:35022

15. H.O. Kreiss, Stability theory of difference approximations for mixed initial boundary value problems (I) Math. Comp., 22 (1968), pp. 703-714. MR39:2355

16. L. Trefethen and L. Halpern, Well posedness of one way wave equations and absorbing boundary conditions, Math. Comp., 47 (1986), pp. 421-435. MR88b:65148

17. D.W. Sheen, Second order absorbing boundary conditions for the wave equation in a rectangular domain, Math. Comp., 61 (1993), pp. 595-606. MR.94f:65089

18. O. Vacus, Singularités de frontières du domaine de calcul: le problème du coin, INRIA Rocquencourt technical report 2851, France, 1996.

CEA/CESTA, 33114 Le Barp Cedex, France

E-mail address: vacus.olivier@wanadoo.fr 